\title{
МАТЕМАТИКА
}

УДК 517.92

MSC $34 \mathrm{C} 14,34 \mathrm{C} 23$

\section{О НЕКОТОРЫХ ЛОКАЛЬНЫХ БИФУРКАЦИЯХ ОБРАТИМЫХ КУСОЧНО-ГЛАДКИХ ДИНАМИЧЕСКИХ СИСТЕМ НА ПЛОСКОСТИ}

\author{
В. Ш. Ройтенберг \\ (Статья представлена членом редакционной коллегии Ю. П. Вирченко) \\ Ярославский государственный технический университет, \\ Ярославль, 150023, Россия \\ E-mail: vroitenberg@mailru
}

\begin{abstract}
Аннотация. Рассматривается двухпараметрическое семейство кусочно-гладких векторных полей, заданных на плоскости, «сшитых» из гладких векторных полей, определенных в верхней и нижней полуплоскостях. Векторные поля семейства являются обратимыми относительно инверсии, для которой линия переключения поля $=0$ состоит из неподвижных точек. При нулевых значениях параметров векторные поля, определенные в верхней и нижней полуплоскостях, имеют в начале координат О касание третьего порядка с осью $x$. Описаны бифуркации фазовых портретов в окрестности точки О при значениях параметров близких к нулю.
\end{abstract}

Ключевые слова: кусочно-гладкое векторное поле, обратимая динамическая система, бифуркация, особая точка, сепаратриса, периодическая траектория.

Для цитирования: Ройтенберг В. Ш. 2021. О некоторых локальных бифуркациях обратимых кусочно-гладких динамических систем на плоскости. Прикладная математика \& Физика. 53(4): 257-265. DOI 10.52575/2687-0959-202153-4-257-265.

\section{ON SOME LOCAL BIFURCATIONS OF REVERSIBLE PIECEWISE SMOOTH DYNAMICAL SYSTEMS ON THE PLANE}

\author{
Vladimir Roitenberg \\ (Article submitted by a member of the editorial board Yu. P. Virchenko) \\ Yaroslavl State Technical University, \\ Yaroslavl, 150023, Russia \\ E-mail: vroitenberg@mail.ru \\ Received November, 21, 2021
}

\begin{abstract}
We consider a two-parameter family of piecewise-smooth vector fields on the plane, "sewn"from smooth vector fields defined in the upper and lower half-planes. The vector fields of the family are assumed to be reversible with respect to an inversion for which the switching line of the field $y=0$ consists of fixed points. At zero values of the parameters, the vector fields defined in the upper and lower half-planes have a third-order tangency with the $x$-axis at the origin of coordinates $\mathrm{O}$. Bifurcations of phase portraits in a neighborhood of point $\mathrm{O}$ are described for parameter values close to zero.
\end{abstract}

Key words: piecewise smooth vector field, reversible dynamical system, bifurcation, singular point, separatrix, periodic trajectory.

For citation: Roitenberg V. Sh. 2021. On some local bifurcations of reversible piecewise smooth dynamical systems on the plane. Applied Mathematics \& Physics. 53(4): 257-265. (in Russian) DOI 10.52575/2687-0959-2021-53-4-257-265.

1. Введение. Динамическая система называется обратимой, если она инвариантна относительно инволюции и обращения времени. Обратимые динамические системы естественно возникают при описании ряда физических процессов [13]. Обратимые системы изучались с разных точек зрения, см., например, $[2,14,15]$. В статье [3] дан обзор работ по локальным бифуркациям гладких обратимых систем. Локальным бифуркациям кусочно-гладких динамических систем посвящено много работ, в частности $[4,7,9,10,11,12]$. Представляет интерес и изучение бифуркаций обратимых кусочно-гладких динамических систем. В работах [5, 6] рассматривались обратимые кусочно-гладкие системы на плоскости, для которых линия разрыва векторного поля, задающего динамическую систему, совпадает с множеством неподвижных точек соответствующей инволюции. Были описаны локальные бифуркации в типичных однопараметрических семействах таких систем. 
В этой работе мы рассмотрим два вида локальных бифуркаций в типичных двухпараметрических семействах обратимых кусочно-гладких векторных полей на плоскости.

2. Обратимые кусочно-гладкие динамические системы на плоскости и их траектории. Пусть $M$ - окрестность точки $O=(0,0)$ на координатной плоскости $\mathbb{R}^{2}$, заданная неравенством $x^{2}+y^{2}<1$, $\mathcal{D}=\left(M_{+}, M_{-}\right)$- ее разбиение на множества $M_{+}$и $M_{-}$, определяемые, соответственно, неравенствами $y \geq 0$ и $y \leq 0$. Пусть $X^{+}$и $X^{-}$- векторные поля на $M$ класса $C^{r}(r \geq 1)$. Кусочно-гладким векторным полем $X=\left(X^{+}, X^{-}\right)$на $M$ с разбиением $\mathcal{D}$ называется класс всех векторных полей $X_{*}$ на $M$ таких, что $X_{*}(x, y)=$ $X^{+}(x, y)$, если $y>0$, и $X_{*}(x, y)=X^{-}(x, y)$, если $y<0$. Множество таких векторных полей обозначим $\mathfrak{X}^{r}(M, \mathcal{D})$. Траектории поля $X=\left(X^{+}, X^{-}\right)$определим согласно [7] как траектории дифференциального включения $\dot{z} \in \hat{X}(z)$, где $\hat{X}(z)=\left\{X^{+}(z)\right\}$, если $z \in \operatorname{int} M_{+}, \hat{X}(z)=\left\{X^{-}(z)\right\}$, если $z \in \operatorname{int} M_{-}$, и $\hat{X}(z)-$ выпуклая оболочка векторов $X^{+}(z)$ и $X^{-}(z)$, если $z \in M_{0}:=M_{+} \cap M_{-}$.

Векторное поле $X=\left(X^{+}, X^{-}\right) \in \mathfrak{X}^{r}(M, \mathcal{D})$, где $X^{ \pm}(x, y)=\left(P^{ \pm}(x, y), Q^{ \pm}(x, y)\right)$, называется обратимым (относительно инверсии $I:(x, y) \mapsto(x,-y))[5,6]$, если

$$
P^{-}(x, y)=-P^{+}(x,-y), \quad Q^{-}(x, y)=Q^{+}(x,-y) .
$$

Множество таких векторных полей будем обозначать $\mathfrak{X}_{I}^{r}(M, \mathcal{D})$. Из (1) следует, что если $L$ - дуга траектории векторного поля $\left.X^{+}\right|_{M_{+}}$, то $I(L)$ - дуга траектории векторного поля $\left.X^{+}\right|_{M_{-}}$.

Точка $z_{0}=\left(x_{0}, 0\right) \in M_{0}$ называется особой точкой поля $X=\left(X^{+}, X^{-}\right) \in \mathfrak{X}_{I}^{r}(M, \mathcal{D})$, если $Q^{+}\left(x_{0}, 0\right)=0$. Особая точка является траекторией поля. Если на $M_{0}$ поле имеет конечное число особых точек, то любая его траектория $L$ либо является траекторией одного из векторных полей $X^{+} \mid M_{+}$или $X^{-} \mid M_{-}$, либо «сшивается» из дуг траекторий этих векторных полей: $L=\{z=\zeta(t): t \in J\}$, где $\zeta: J \rightarrow M-$ непрерывная функция на промежутке $J$ (открытом, полуоткрытом или замкнутом), причем для всех $t \in J$, кроме их конечного числа, $\zeta(t) \in M_{+}$и $\dot{\zeta}(t)=X^{+}(\zeta(t))$ или $\zeta(t) \in M_{-}$и $\dot{\zeta}(t)=X^{-}(\zeta(t))$ или $\zeta(t)-$ особая точка и $\dot{\zeta}(t)=0$. При этом уравнение $z=I(\zeta(-t))$ также задает траекторию поля $X$.

Особая точка $z_{0}=\left(x_{0}, 0\right)$ называется квазиседлом, если $P^{+}\left(z_{0}\right)\left(Q^{+}\right)_{x}^{\prime}\left(z_{0}\right)>0$, и квазицентром, если $P^{+}\left(z_{0}\right)\left(Q^{+}\right)_{x}^{\prime}\left(z_{0}\right)<0[5,6]$. Через квазиседло проходят траектории векторных полей $\left.X^{+}\right|_{M_{+}}$и $\left.X^{-}\right|_{M_{-}}$, касающиеся в этой точке $M_{0}$. Их положительные (отрицательные) полутраектории, начинающиеся в точке $z_{0}$, будем называть выходящими (входящими) сепаратрисами квазиседла. Сепаратрисы разбивают окрестность квазиседла на четыре гиперболических сектора (рис. 1а). Квазицентр является траекторией поля $X$, а все остальные траектории, проходящие через точки некоторой его окрестности, являются замкнутыми (периодическими) (рис. 1б).

Особую точку $z_{0}$ назовем клювом, если $Q^{+}\left(z_{0}\right)=\left(Q^{+}\right)_{x}^{\prime}\left(z_{0}\right)=0, P^{+}\left(z_{0}\right)\left(Q^{+}\right)_{x x}^{\prime \prime}\left(z_{0}\right) \neq 0$. Пусть для определенности $P^{+}\left(z_{0}\right)\left(Q^{+}\right)_{x x}^{\prime \prime}\left(z_{0}\right)>0$. Траектория поля $\left.X^{+}\right|_{M_{+}}\left(\left.X^{-}\right|_{M_{-}}\right)$, начинающаяся в точке $z_{0}$, совпадает со своей положительной (отрицательной) полутраекторией и касается $M_{0}$ в этой точке. Будем называть ее выходящей (входящей) сепаратрисой клюва. Сепаратрисы разбивают окрестность клюва на два гиперболических сектора (рис. 1в). Случай $P^{+}\left(z_{0}\right)\left(Q^{+}\right)_{x x}^{\prime \prime}\left(z_{0}\right)<0$ сводится к рассмотренному случаю переходом к векторному полю $-X$. Бифуркации клюва рассматривались в работе [6].

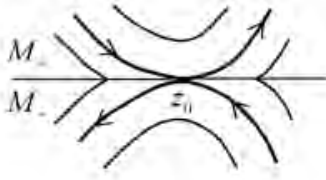

a)



6)



R)

Рис. 1. Особые точки: а) квазиседло, б) квазицентр, в) клюв

Fig. 1. Singular points: a) quasisaddle, б) quasicenter, в) beak

3. Основные результаты. Рассмотрим семейство векторных полей $X_{\varepsilon}=\left(X_{\varepsilon}^{+}, X_{\varepsilon}^{-}\right) \in \mathfrak{X}_{I}^{r}(M, \mathcal{D})$, зависящих от параметра $\varepsilon$, меняющегося в некоторой окрестности Е' нуля двумерного пространства Е. Пусть $X_{\varepsilon}^{ \pm}(x, y)=\left(P^{ \pm}(x, y, \varepsilon), Q^{ \pm}(x, y, \varepsilon)\right)$, где $P_{i}^{ \pm}, Q_{i}^{ \pm}(i=1,2)-C^{r}$-функции, $r \geq 4$. Будем предполагать, что выполняются следующие условия:

Условие 1. $P^{+}(0)>0, Q^{+}(0)=\left(Q^{+}\right)_{x}^{\prime}(0)=\left(Q^{+}\right)_{x x}^{\prime \prime}(0)=0,\left(Q^{+}\right)_{x x x}^{\prime \prime \prime}(0) \neq 0,\left(Q^{+}\right)_{y}^{\prime}(0) \neq 0$.

Условие 2. Ковекторы $\left(Q^{+}\right)_{\varepsilon}^{\prime}(0)$ и $\left(Q^{+}\right)_{x \varepsilon}^{\prime \prime}(0)$ линейно независимы.

Из условия 1 следует, что для векторного поля $X_{0}$ поведение траектории в окрестности особой точки $O=(0,0)$ при $\left(Q^{+}\right)_{x x x}^{\prime \prime \prime}(0)>0$ аналогично поведению траекторий в окрестности квазиседла, отличие только в большем порядке касания сепаратрис с $M_{0}$, а при $\left(Q^{+}\right)_{x x x}^{\prime \prime \prime}(0)<0$ аналогично поведению траекторий в окрестности квазицентра.

Без ограничения общности можно считать $\left(Q^{+}\right)_{y}^{\prime}(0)<0$. Если $\left(Q^{+}\right)_{y}^{\prime}(0)>0$, то следует заменить векторные поля $X_{\varepsilon}$ на векторные поля $-X_{\varepsilon}$, а координату $x$ на $-x$. 
Выберем число $d_{0}>0$ и окрестности $\mathrm{E}^{\prime \prime}$ нуля в Е так, чтобы $P^{+}(x, y, \varepsilon)>0$ при $|x|<d_{0},|y|<d_{0}$, $\varepsilon \in \mathrm{E}^{\prime \prime}$. Тогда траектории поля $X_{\varepsilon}^{+}, \varepsilon \in \mathrm{E}^{\prime \prime}$, в области $|x|<d_{0},|y|<d_{0}$ являются интегральными кривыми дифференциального уравнения $\frac{d y}{d x}=R(x, y, \varepsilon)$, где $R=Q^{+} / P^{+}$. Из условия 1 имеем

$$
\begin{gathered}
R(0)=R_{x}^{\prime}(0)=R_{x x}^{\prime \prime}(0)=0, \quad \operatorname{sgn} R_{x x x}^{\prime \prime \prime}(0)=\operatorname{sgn}\left(Q^{+}\right)_{x x x}^{\prime \prime \prime}(0), \\
R_{y}^{\prime}(0)<0
\end{gathered}
$$

Определим функции $f_{i}: \mathrm{E}^{\prime \prime} \rightarrow \mathbb{R}$, положив

$$
f_{1}(\varepsilon):=-R_{x}^{\prime}(0,0, \varepsilon) \cdot \operatorname{sgn} R_{x x x}^{\prime \prime \prime}(0), \quad f_{2}(\varepsilon):=R(0,0, \varepsilon) .
$$

Так как $R_{\varepsilon}^{\prime}(0)=Q_{\varepsilon}^{\prime}(0) / P(0), R_{x \varepsilon}^{\prime \prime}(0)=Q_{x \varepsilon}^{\prime \prime}(0) / P(0)-P_{x}^{\prime}(0) Q_{\varepsilon}^{\prime}(0) / P^{2}(0)$, то из условия 2 и (4) следует, что и ковекторы $\left(f_{2}\right)_{\varepsilon}^{\prime}(0)$ и $\left(f_{1}\right)_{\varepsilon}^{\prime}(0)$ линейно независимы. Поэтому в некоторой окрестности $\mathrm{E}_{0}$ нуля в $\mathrm{E}$ существуют такие $C^{r-1}$-координаты $\varepsilon_{1}, \varepsilon_{2}$, что

$$
\forall \varepsilon \in \mathrm{E}_{0} \quad f_{1}(\varepsilon)=\varepsilon_{1}, \quad f_{2}(\varepsilon)=\varepsilon_{2} .
$$

Далее будем отождествлять точку $\varepsilon \in \mathrm{E}_{0}$ с ее координатной строкой: $\varepsilon=\left(\varepsilon_{1}, \varepsilon_{2}\right)$ и полагать $\mathrm{E}_{0}=$ $\left(-\delta_{0}, \delta_{0}\right)^{2}$ при некотором $\delta_{0}>0$.

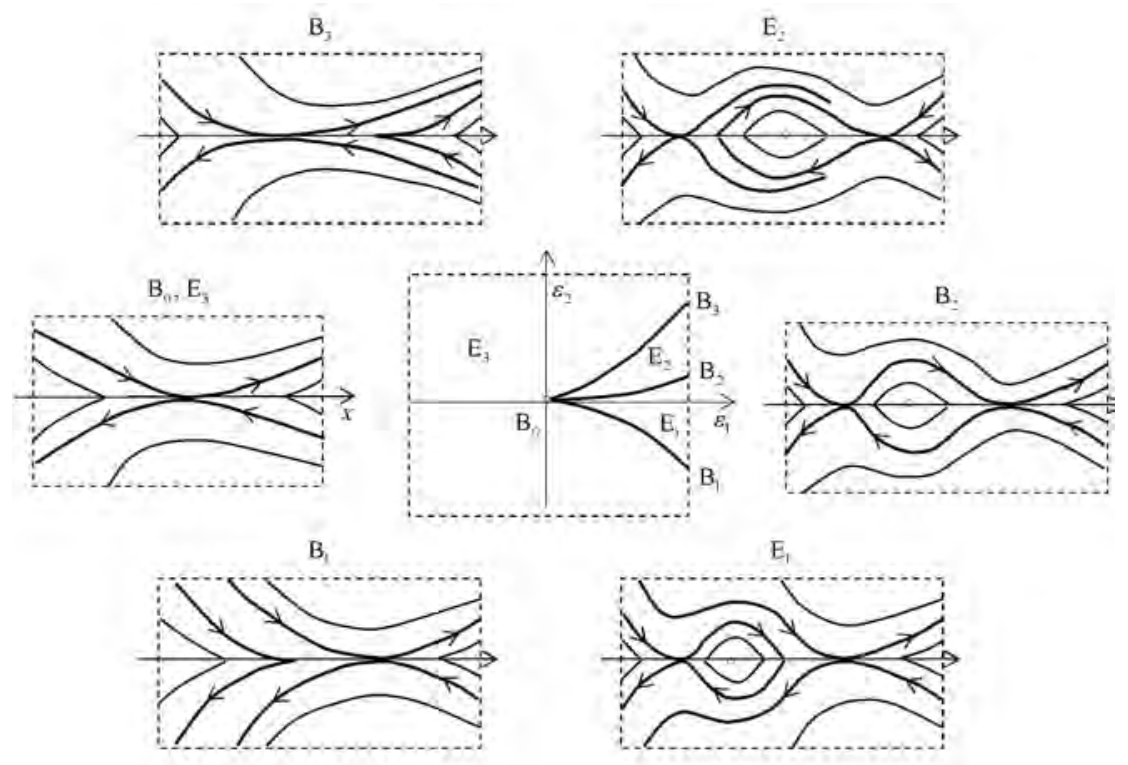

Рис. 2. Бифуркационная диаграмма в случае $\left(Q^{+}\right)_{x x x}^{\prime \prime \prime}(0)>0$

Fig. 2. Bifurcation diagram in the case $\left(Q^{+}\right)_{x x x}^{\prime \prime \prime}(0)>0$

Теорема 1. Пусть для семейства векторных полей $X_{\varepsilon} \in \mathfrak{X}_{I}^{r}(M, \mathcal{D})$ выполняются условия 1 и 2 , а $\left(Q^{+}\right)_{x х x}^{\prime \prime \prime}(0)>0$. Тогда существуют окрестность $U$ точки $O$, число $\delta \in\left(0, \delta_{0}\right)$ и разбиение области параметров $(-\delta, \delta)^{2}$ на множества $B_{0}=\{(0,0)\}, E_{k}, B_{k}, k=1,2,3$, где

$B_{k}=\left\{\varepsilon: \varepsilon_{2}=b_{k}\left(\varepsilon_{1}\right)\right\}, b_{k}:(0, \delta) \rightarrow(-\delta, \delta), b_{k} \in C^{1}, b_{1}(+0)=b_{1}^{\prime}(+0)=b_{3}(+0)=b_{3}^{\prime}(+0)=0, \forall \varepsilon_{1} \in(0, \delta)$ $b_{1}\left(\varepsilon_{1}\right)<0, b_{3}\left(\varepsilon_{1}\right)>0, b_{1}\left(\varepsilon_{1}\right)<b_{2}\left(\varepsilon_{1}\right)<b_{3}\left(\varepsilon_{1}\right)$,

$E_{k}$ - связная компонента $(-\delta, \delta)^{2} \backslash \bigcup_{i=0}^{3} B_{i}$, в границу которой входят $B_{k}$ и $B_{k+1}\left(B_{4}:=B_{1}\right)$, со следующими свойствами:

Фазовые портреты векторных полей $X_{\varepsilon}$ в $U$ при $\varepsilon \in E_{k}$ и $\varepsilon \in B_{k}$ имеют вид, изображенный на рис. 2.

Если $\varepsilon \in B_{1}$ или $\varepsilon \in B_{3}$, то $X_{\varepsilon}$ имеет в $U$ две особые точки: квазиседло и клюв. Выходящие (входящие) сепаратрисы квазиседла при возрастании (убывании) времени покидают U. При $\in \in B_{1}$ входящая (выходящая) сепаратриса клюва принадлежит $M_{+}\left(M_{-}\right)$и при убывании (возрастании) времени покидает U. При $\varepsilon \in B_{3}$ входящая (выходящая) сепаратриса клюва принадлежит $M_{-}\left(M_{+}\right)$, и при убыввании возрастании (возрастании) времени покидает U. Все остальные траектории выходят из U и при возрастании, и при убывании времени.

Если $\varepsilon \in B_{2} \cup E_{1} \cup E_{2}$, то $X_{\varepsilon}$ имеет в $U$ три особых точки: квазиседла $z_{ \pm}(\varepsilon)=\left(x_{ \pm}(\varepsilon), 0\right)$ и квазицентр $z_{0}(\varepsilon)=\left(x_{0}(\varepsilon), 0\right)$, где $x_{-}(\varepsilon)<x_{0}(\varepsilon)<x_{+}(\varepsilon)$.

При $\varepsilon \in B_{2}$ выходящая (входящая) сепаратриса квазиседла $z_{+}(\varepsilon)$ совпадает с входящей (выходящей) сепаратрисой квазиседла $z_{-}(\varepsilon)$. Эти сепаратрисы образуют контур, являющийся периодической траекторией и ограничивающий вместе с квазицентром $z_{0}(\varepsilon)$ кольщевую область, состоящую из периодических траекторий. Две другие выходящие (входящие) сепаратрисы точек $z_{ \pm}(\varepsilon)$ при возрастании (убьвании) времени покидают U. Все остальные траектории выходят из U и при возрастании и при убьвании времени. 
При $\varepsilon \in E_{1}$, соотв. при $\varepsilon \in E_{2}$, выходящзая и входящая сепаратриса квазиседла $z_{-}(\varepsilon)$, соотв. $z_{+}(\varepsilon)$, образуют контур, являющийся периодической траекторией, и ограничивающий вместе с квазицентром $z_{0}(\varepsilon)$ кольцевую область, состоящую из периодчческих траекторий. Другая выходящая (входящая) сепаратриса этой точки, а также выходящие (входящие) сепаратрисы точки $z_{+}(\varepsilon)$ соотв. точки $z_{-}(\varepsilon)$, при возрастании (убывании) времени покидают U. Все остальные траектории выходят из $U$ и при возрастании и при убьввании времени.

Если $\varepsilon \in E_{3}$, то $X_{\varepsilon}$ имеет в $U$ единственную особую точку - квазиседло, выходящце (входящие) сепаратрисы которого при возрастании (убывании) времени покидают U. Все остальные траектории выходят из U и при возрастании, и при убывании времени.

При любых $\varepsilon^{\prime}, \varepsilon^{\prime \prime} \in E_{k}\left(\varepsilon^{\prime}, \varepsilon^{\prime \prime} \in B_{k}\right)$ векторные поля $X_{\varepsilon^{\prime}} u X_{\varepsilon^{\prime \prime}}$ топологически эквивалентны в $U$.

Доказательство теоремы 1 приведено в разделах 4-6.

Теорема 2. Пусть семейство векторных полей $X_{\varepsilon} \in \mathfrak{X}_{I}^{r}(M, \mathcal{D})$ удовлетворяет условиям 1 и 2 и $\left(Q^{+}\right)_{x х x}^{\prime \prime \prime}(0)<0$. Тогда существуют окрестность $U$ точки $O$, число $\delta \in\left(0, \delta_{0}\right)$ и разбиение области параметров $(-\delta, \delta)^{2}$ на множества $B_{0}=\{(0,0)\}, E_{k}, B_{k}, k=1,2$, где

$B_{k}=\left\{\varepsilon: \varepsilon_{2}=b_{k}\left(\varepsilon_{1}\right)\right\}, b_{k}:(0, \delta) \rightarrow(-\delta, \delta), b_{k} \in C^{1}, b_{k}(+0)=b_{k}^{\prime}(+0)=0, b_{1}\left(\varepsilon_{1}\right)<0<b_{3}\left(\varepsilon_{1}\right)$,

$E_{k}$ - связная компонента множества $(-\delta, \delta)^{2} \backslash\left(B_{0} \cup B_{1} \cup B_{2}\right)$, в гранииу которой входят $B_{k} u B_{k+1}\left(B_{3}:=B_{1}\right)$, со следующими свойствами:

Фазовые портреты векторных полей $X_{\varepsilon}$ в $U$ при $\varepsilon \in E_{k}$ и $\varepsilon \in B_{k}$ имеют вид, изображенный на рис. 3.

При любых $\varepsilon^{\prime}, \varepsilon^{\prime \prime} \in E_{k}\left(\varepsilon^{\prime}, \varepsilon^{\prime \prime} \in B_{k}\right)$ векторные поля $X_{\varepsilon^{\prime}} u X_{\varepsilon^{\prime \prime}}$ топологически эквивалентны в $U$.

Доказательство теоремы 2 аналогично доказательству теоремы 1 и потому опускается.

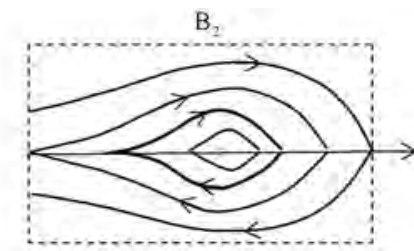

$\mathrm{B}_{0+} \mathrm{E}$.
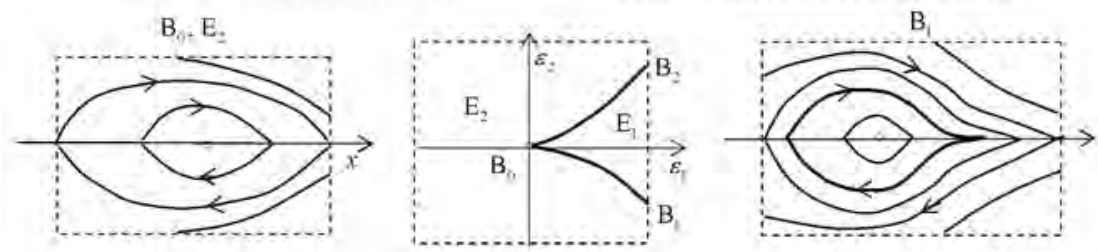

Рис.3. Бифуркационная диаграмма в случае $\left(Q^{+}\right)_{x x x}^{\prime \prime \prime}(0)<0$

Fig. 3. Bifurcation diagram in the case $\left(Q^{+}\right)_{x x x}^{\prime \prime \prime}(0)<0$

4. Окрестность $U$. Особые точки. Бифуркационные кривые $B_{1}$ и $B_{3}$. По условию теоремы $\left(Q^{+}\right)_{x x x}^{\prime \prime \prime}(0)>0$. Ввиду (2) и (3) можно выбрать такое число $d_{1}>0$, что $R\left(0, d_{1}, 0\right)<0$. Отсюда и из (2) следует, что, взяв достаточно малые $d_{2}>0$ и $\delta_{1} \in\left(0, \delta_{0}\right)$, будем иметь при всех $x \in\left[-d_{2}, d_{2}\right], \varepsilon \in\left[-\delta_{1}, \delta_{1}\right]^{2}$

$$
\begin{gathered}
R\left(x, d_{1}, \varepsilon\right)<0, \\
R_{x x x}^{\prime \prime \prime}(x, 0, \varepsilon)>0, \\
\pm R\left( \pm d_{2}, 0, \varepsilon\right)>0 .
\end{gathered}
$$

Зададим окрестность $U$ точки $O$ неравенствами $|x|<d_{2},|y|<d_{1}$. Ее граница $\partial U$ является объединением дуг $\gamma_{i}^{ \pm}(i=1,2,3)$, где $\gamma_{1}^{+}=\left\{(x, y): x=-d_{2}, 0 \leq y \leq d_{1}\right\}, \gamma_{2}^{+}=\left\{(x, y):-d_{2} \leq x \leq d_{2}, y=d_{1}\right\}$, $\gamma_{3}^{+}=\left\{(x, y): x=d_{2}, 0 \leq y \leq d_{1}\right\}, \gamma_{i}^{-}=I\left(\gamma_{i}^{+}\right)$. Вследствие (6) $\forall \varepsilon \in\left(-\delta_{1}, \delta_{1}\right)^{2}$ траектории векторного поля $X_{\varepsilon}^{+}\left(X_{\varepsilon}^{-}\right)$трансверсальны дугам $\gamma_{i}^{+}\left(\gamma_{i}^{-}\right)$, причем во внутренних точках $\gamma_{1}^{+}$и $\gamma_{2}^{+}\left(\gamma_{1}^{-}\right.$и $\left.\gamma_{2}^{-}\right)$они входят в $U$ (выходят из $\bar{U})$, а в точках $\gamma_{3}^{+}\left(\gamma_{3}^{-}\right)$они выходят из из $\bar{U}$ (входят в $U$ ).

Вследствие (7) и (8) функция $R(\cdot, 0, \varepsilon)$ имеет на отрезке $\left[-d_{2}, d_{2}\right]$ либо один, либо три нуля с учетом их кратности.

Пусть $Y(x, u, v, \varepsilon)$ - решение уравнения $y^{\prime}=R(x, y, \varepsilon)$, которое удовлетворяет начальному условию $Y(u, u, v, \varepsilon)=v$. Обозначим $N$ наибольшее значение $|R(x, y, \varepsilon)|$ в $\bar{U} \times\left[-\delta_{1}, \delta_{1}\right]^{2}$. Пусть $0<d_{*}<$ $\min \left\{d_{2}, N / 2 d_{2}\right\}$. Тогда при $|u|<d_{*}$ решение $Y(x, u, 0, \varepsilon)$ определено для всех $x \in\left[-d_{2}, d_{2}\right]$.

Вследствие (2) числа $d_{*}$ и $\delta_{2} \in\left(0, \delta_{1}\right)$ можно выбрать так, что все нули функции $R(\cdot, 0, \varepsilon), \varepsilon \in\left(-\delta_{2}, \delta_{2}\right)^{2}$, принадлежат интервалу $\left(-d_{*}, d_{*}\right)$.

Кратные нули функции $R(\cdot, 0, \varepsilon)$ удовлетворяют системе уравнений $R(x, 0, \varepsilon)=R_{x}^{\prime}(x, 0, \varepsilon)=0$. С учетом равенств (2), (4) и (5) ее можно записать в виде

$$
\varepsilon_{2}-\varepsilon_{1} x+a(\varepsilon) x^{2}+c(x, \varepsilon)=0,-\varepsilon_{1}+2 a(\varepsilon) x+c_{x}^{\prime}(x, \varepsilon)=0,
$$


где

$$
a(\cdot) \in C^{1}, a(0)=0, c(\cdot, \cdot) \in C^{4}, c(x, \varepsilon)=\left(R_{x x x}^{\prime \prime \prime}(0, \varepsilon) / 6\right) x^{3}+o\left(x^{3}\right), c_{x}^{\prime}(x, \varepsilon)=\left(R_{x x x}^{\prime \prime \prime}(0, \varepsilon) / 2\right) x^{2}+o\left(x^{2}\right) .
$$

По теореме о неявной функции существует такое $\bar{x} \in\left(0, d_{*}\right)$, что для любого $x \in[-\bar{x}, \bar{x}]$ система (9) имеет относительно $\varepsilon \in\left(-\delta_{2}, \delta_{2}\right)^{2}$ решение $\varepsilon=\tilde{\varepsilon}(x)=\left(\tilde{\varepsilon}_{1}(x), \tilde{\varepsilon}_{2}(x)\right)$, где $\tilde{\varepsilon}_{k}(\cdot)(k=1,2)-C^{3}$-функции, $\tilde{\varepsilon}_{k}(0)=0$. Записав их в виде

$$
\tilde{\varepsilon}_{1}(x)=\alpha_{1} x+\alpha_{2} x^{2}+o\left(x^{2}\right), \quad \tilde{\varepsilon}_{2}(x)=\beta_{1} x+\beta_{2} x^{2}+\beta_{3} x^{3}+o\left(x^{3}\right)
$$

и подставив в (9), получим $\alpha_{1}=\beta_{1}=\beta_{2}=0, \alpha_{2}=R_{x x x}^{\prime \prime \prime}(0) / 2, \beta_{3}=R_{x x x}^{\prime \prime \prime}(0) / 3$. Таким образом, $R(\cdot, 0, \varepsilon)$ имеет кратный нуль $x \in[-\bar{x}, \bar{x}]$ при значениях параметров

$$
\varepsilon_{1}=\tilde{\varepsilon}_{1}(x)=\alpha_{2} x^{2}+o\left(x^{2}\right), \quad \varepsilon_{2}=\tilde{\varepsilon}_{2}(x)=\beta_{3} x^{3}+o\left(x^{3}\right),
$$

где $\alpha_{2}>0, \beta_{3}>0$. Так как

$$
R_{x x}^{\prime \prime}(x, 0, \tilde{\varepsilon}(x))=2 a(\tilde{\varepsilon}(x))+R_{x x x}^{\prime \prime \prime}(0,0, \tilde{\varepsilon}(x)) x+o(x)=R_{x x x}^{\prime \prime \prime}(0) x+o(x)
$$

то $\bar{x}$ можно считать выбранным так, что $\operatorname{sgn} R_{x x}^{\prime \prime}(x, \tilde{\varepsilon}(x))=\operatorname{sgn} x$ при $x \in[-\bar{x}, \bar{x}]$. Следовательно, $x \in[-\bar{x}, \bar{x}], x \neq 0$ - двукратный нуль функции $R(\cdot, 0, \varepsilon)$ при $\varepsilon=\tilde{\varepsilon}(x)$. Ввиду (10) мы можем также считать $\bar{x}$ столь малым, что 1) $\tilde{\varepsilon}_{1}(x)$ убывает на $[-\bar{x}, 0]$, возрастает на $[0, \bar{x}]$ и потому имеет на этих отрезках непрерывные обратные функции, соответственно $\tilde{x}_{-}\left(\varepsilon_{1}\right), \varepsilon_{1} \in\left[0, \tilde{\varepsilon}_{1}(-\bar{x})\right]$ и $\tilde{x}_{+}\left(\varepsilon_{1}\right), \varepsilon_{1} \in\left[0, \tilde{\varepsilon}_{1}(\bar{x})\right]$, дифференцируемые при $\varepsilon_{1} \neq 0$; 2) $\left|\tilde{\varepsilon}_{2}( \pm \bar{x})\right|<\tilde{\varepsilon}_{1}( \pm \bar{x})$. Возьмем $0<\delta<\min \left\{\tilde{\varepsilon}_{1}(-\bar{x}), \tilde{\varepsilon}_{1}(\bar{x})\right\}$ и положим $b_{1}\left(\varepsilon_{1}\right):=\tilde{\varepsilon}_{2}\left(\tilde{x}_{-}\left(\varepsilon_{1}\right)\right), \varepsilon_{1} \in(0, \delta)$ и $b_{3}\left(\varepsilon_{1}\right):=\tilde{\varepsilon}_{2}\left(\tilde{x}_{+}\left(\varepsilon_{1}\right)\right), \varepsilon_{1} \in(0, \delta)$. Из (10) следует, что $b_{k}(+0)=b_{k}^{\prime}(+0)=0$, $k=1,3$.

При $\varepsilon_{2}=b_{1}\left(\varepsilon_{1}\right) R(\cdot, 0, \varepsilon)$ кроме $\tilde{x}_{-}\left(\varepsilon_{1}\right)$ имеет еще один (простой) нуль, который обозначим $x_{+}\left(\varepsilon_{1}\right)$. При $\varepsilon_{2}=b_{1}\left(\varepsilon_{1}\right) R(x, 0, \varepsilon)=R_{x x}^{\prime \prime}\left(\tilde{x}_{-}\left(\varepsilon_{1}\right), \varepsilon\right)\left(x-\tilde{x}_{-}\left(\varepsilon_{1}\right)\right)^{2}+o\left(\left(x-\tilde{x}_{-}\left(\varepsilon_{1}\right)\right)^{2}\right)$. Поэтому $R\left(x_{*}, 0, \varepsilon\right)<0$ при некотором $x_{*}>\tilde{x}_{-}\left(\varepsilon_{1}\right)$. Отсюда и из (8) получаем что $\tilde{x}_{-}\left(\varepsilon_{1}\right)<x_{+}\left(\varepsilon_{1}\right)<d$ и

$$
\begin{gathered}
R(x, 0, \varepsilon)<0 \text { при } x \in\left[-d, \tilde{x}_{-}\left(\varepsilon_{1}\right)\right) \cup\left(\tilde{x}_{-}\left(\varepsilon_{1}\right), x_{+}\left(\varepsilon_{1}\right)\right), R(x, 0, \varepsilon)>0 \text { при } x \in\left(x_{+}\left(\varepsilon_{1}\right), d\right], \\
R_{x}^{\prime}\left(x_{+}\left(\varepsilon_{1}\right), 0, \varepsilon\right)>0 .
\end{gathered}
$$

Аналогично, при $\varepsilon_{2}=b_{3}\left(\varepsilon_{1}\right)$ функция $R(\cdot, 0, \varepsilon)$ кроме $\tilde{x}_{+}\left(\varepsilon_{1}\right)$ имеет еще один (простой) нуль $x_{-}\left(\varepsilon_{1}\right)$; при этом $-d<x_{-}\left(\varepsilon_{1}\right)<\tilde{x}_{+}\left(\varepsilon_{1}\right)$,

$$
\begin{gathered}
R(x, 0, \varepsilon)<0 \text { при } x \in\left[-d, x_{-}\left(\varepsilon_{1}\right)\right), R(x, 0, \varepsilon)>0 \text { при } x \in\left(x_{-}\left(\varepsilon_{1}\right), \tilde{x}_{+}\left(\varepsilon_{1}\right)\right) \cup\left(\tilde{x}_{+}\left(\varepsilon_{1}\right), d\right], \\
R_{x}^{\prime}\left(x_{-}\left(\varepsilon_{1}\right), 0, \varepsilon\right)>0 .
\end{gathered}
$$

Пусть $B_{k}:=\left\{\varepsilon: \varepsilon_{2}=b_{k}\left(\varepsilon_{1}\right)\right\}, k=1,3$.

5. Бифуркационная кривая $B_{2}$. Обозначим $B_{*}:=\left\{\varepsilon: 0<\varepsilon_{1}<\delta, b_{1}\left(\varepsilon_{1}\right)<\varepsilon_{2}<b_{3}\left(\varepsilon_{1}\right)\right\}$. Так как при $\varepsilon_{2}=0$ точка $x=0$ - нуль функции $R(x, 0, \varepsilon)=-\varepsilon_{1} x+a(\varepsilon) x^{2}+o\left(x^{2}\right)$ и $R_{x}^{\prime}(0,0, \varepsilon)=-\varepsilon_{1}<0$, то с учетом (8) получаем, что имеется еще два простых нуля. Так как простые нули непрерывно зависят от параметра, а $B_{*}$ - связное множество, то $\forall \varepsilon \in B_{*} R(\cdot, 0, \varepsilon)$ имеет на $(-d, d)$ три простых нуля

$$
x_{-}(\varepsilon)<x_{0}(\varepsilon)<x_{+}(\varepsilon) .
$$

По теореме о неявной функции $x_{-}(\cdot), x_{0}(\cdot), x_{+}(\cdot) \in C^{1}$. Из (8) следует, что

$$
\begin{aligned}
& R(x, 0, \varepsilon)>0 \text { при } x \in\left(x_{-}(\varepsilon), x_{0}(\varepsilon)\right) \cup\left(x_{+}(\varepsilon), d_{2}\right), \\
& R(x, 0, \varepsilon)<0 \text { при } x \in\left(-d_{2}, x_{-}(\varepsilon)\right) \cup\left(x_{0}(\varepsilon), x_{+}(\varepsilon)\right),
\end{aligned}
$$

$R_{x}^{\prime}\left(x_{ \pm}(\varepsilon), 0, \varepsilon\right)>0, \quad R_{x}^{\prime}\left(x_{0}(\varepsilon), 0, \varepsilon\right)<0$, и потому точки $z_{ \pm}(\varepsilon)=\left(x_{ \pm}\left(\varepsilon_{1}\right), 0\right)-$ квазиседла, а точка $z_{0}(\varepsilon)=$ $\left(x_{0}\left(\varepsilon_{1}\right), 0\right)$ - квазицентр.

Производная $Y_{\varepsilon_{2}}^{\prime}(x, u, v, \varepsilon)$ решения $Y(x, u, v, \varepsilon)$ уравнения $y^{\prime}=R(x, y, \varepsilon)$ удовлетворяет уравнению в вариациях

$$
\frac{d}{d x} Y_{\varepsilon_{2}}^{\prime}=R_{y}^{\prime}(x, Y(x, u, v, \varepsilon), \varepsilon) Y_{\varepsilon_{2}}^{\prime}+R_{\varepsilon_{2}}^{\prime}(x, Y(x, u, v, \varepsilon), \varepsilon)
$$

и начальному условию $Y_{\varepsilon_{2}}^{\prime}(u, u, v, \varepsilon)=0$. Следовательно,

$$
Y_{\varepsilon_{2}}^{\prime}(x, u, v, \varepsilon)=\int_{u}^{x} R_{\varepsilon_{2}}^{\prime}(s, Y(s, u, v, \varepsilon), \varepsilon) \exp \int_{s}^{x} R_{y}^{\prime}(\xi, Y(\xi, u, v, \varepsilon), \varepsilon) d \xi d s
$$


Функции $Y\left(\cdot, x_{ \pm}(\varepsilon), 0, \varepsilon\right)$ определены во всех точках отрезка $\left[-d_{2}, d_{2}\right]$. Так как $R(0,0, \varepsilon)=\varepsilon_{2}$, то $R_{\varepsilon_{2}}^{\prime}(0,0, \varepsilon)=1$, и потому окрестность $U$ и $\delta$ можно считать столь малыми, что $R_{\varepsilon_{2}}^{\prime}(x, y, \varepsilon) \geq 1 / 2$ при всех $(x, y) \in U$, $\varepsilon \in[-\delta, \delta]^{2}$. Теперь из (15) и (17) имеем

$$
Y_{\varepsilon_{2}}^{\prime}\left(0, x_{-}(\varepsilon), 0, \varepsilon\right)>0, Y_{\varepsilon_{2}}^{\prime}\left(0, x_{+}(\varepsilon), 0, \varepsilon\right)<0 .
$$

Определим функцию $\Delta(\varepsilon):=Y\left(0, x_{-}(\varepsilon), 0, \varepsilon\right)-Y\left(0, x_{+}(\varepsilon), 0, \varepsilon\right)$. Ее производная

$$
\Delta_{\varepsilon_{2}}^{\prime}(\varepsilon)=Y_{u}^{\prime}\left(0, x_{-}(\varepsilon), 0, \varepsilon\right)\left(x_{-}\right)_{\varepsilon_{2}}^{\prime}(\varepsilon)+Y_{\varepsilon_{2}}^{\prime}\left(0, x_{-}(\varepsilon), 0, \varepsilon\right)-Y_{u}^{\prime}\left(0, x_{+}(\varepsilon), 0, \varepsilon\right)\left(x_{+}\right)_{\varepsilon_{2}}^{\prime}(\varepsilon)-Y_{\varepsilon_{2}}^{\prime}\left(0, x_{+}(\varepsilon), 0, \varepsilon\right) .
$$

По формуле для производной решения дифференциального уравнения по начальному значению аргумента $\left[8\right.$, с. 120] $Y_{u}^{\prime}\left(0, x_{ \pm}(\varepsilon), 0, \varepsilon\right)=-Y_{v}^{\prime}\left(0, x_{ \pm}(\varepsilon), 0, \varepsilon\right) R\left(x_{ \pm}(\varepsilon), 0, \varepsilon\right)=0$. Отсюда, из (18) и (19) получаем

$$
\Delta_{\varepsilon_{2}}^{\prime}(\varepsilon)>0 \text {. }
$$

Фиксируем $\varepsilon_{1} \in(0, \delta)$. Пусть $\varepsilon=\hat{\varepsilon}(\mu):=\left(\varepsilon_{1}, b_{1}\left(\varepsilon_{1}\right)+\mu\right)$. При достаточно малом $\mu>0 x_{-}(\hat{\varepsilon}(\mu))<$ $x_{0}(\hat{\varepsilon}(\mu))<0<x_{+}(\hat{\varepsilon}(\mu))$, причем

$$
\begin{gathered}
\lim _{\mu \rightarrow 0} x_{-}(\hat{\varepsilon}(\mu))=\lim _{\mu \rightarrow 0} x_{0}(\hat{\varepsilon}(\mu))=\tilde{x}_{-}\left(\varepsilon_{1}\right), \\
\lim _{\mu \rightarrow 0} x_{+}(\hat{\varepsilon}(\mu))=\tilde{x}_{+}\left(\varepsilon_{1}\right) .
\end{gathered}
$$

Ввиду (11)

$$
Y\left(\tilde{x}_{-}\left(\varepsilon_{1}\right), x_{+}\left(\varepsilon_{1}\right), 0, \hat{\varepsilon}(0)\right)>0 .
$$

Вследствие (21) - (23) при достаточно малых $\mu$

$$
Y\left(x_{0}(\hat{\varepsilon}(\mu)), x_{+}(\hat{\varepsilon}(\mu)), 0, \hat{\varepsilon}(\mu)\right)>Y\left(\tilde{x}_{-}\left(\varepsilon_{1}\right), x_{+}\left(\varepsilon_{1}\right), 0, \hat{\varepsilon}(0)\right) / 2>0 .
$$

Из (21) следует, что $\lim _{\mu \rightarrow 0} Y\left(x_{0}(\hat{\varepsilon}(\mu)), x_{-}(\hat{\varepsilon}(\mu)), 0, \hat{\varepsilon}(\mu)\right)=Y\left(\tilde{x}_{-}\left(\varepsilon_{1}\right), \tilde{x}_{-}\left(\varepsilon_{1}\right), 0, \hat{\varepsilon}(0)\right)=0$. Отсюда и из (24) получаем, что про достаточно малых $\mu$

$$
Y\left(x_{0}(\hat{\varepsilon}(\mu)), x_{-}(\hat{\varepsilon}(\mu)), 0, \hat{\varepsilon}(\mu)\right)<Y\left(x_{0}(\hat{\varepsilon}(\mu)), x_{+}(\hat{\varepsilon}(\mu)), 0, \hat{\varepsilon}(\mu)\right) .
$$

Но тогда и $Y\left(0, x_{-}(\hat{\varepsilon}(\mu)), 0, \hat{\varepsilon}(\mu)\right)<Y\left(0, x_{+}(\hat{\varepsilon}(\mu)), 0, \hat{\varepsilon}(\mu)\right)$. Тем самым,

$$
\Delta\left(\varepsilon_{1}, b_{1}\left(\varepsilon_{1}\right)+\mu\right)<0 .
$$

Аналогично, используя (13), доказывается, что достаточно малых $\mu>0$

$$
\Delta\left(\varepsilon_{1}, b_{3}\left(\varepsilon_{1}\right)-\mu\right)>0 .
$$

Из $(25),(26)$ и (20) получаем, что существует число $b_{2}\left(\varepsilon_{1}\right) \in\left(b_{1}\left(\varepsilon_{1}\right), b_{3}\left(\varepsilon_{1}\right)\right)$ такое, что

$$
\operatorname{sgn} \Delta(\varepsilon)=\operatorname{sgn}\left(\varepsilon_{2}-b_{2}\left(\varepsilon_{1}\right)\right) \text {. }
$$

Из (27) и (20) по теореме о неявной функции следует, что $b_{2}(\cdot) \in C^{1}$. Положим $B_{2}:=\left\{\varepsilon: \varepsilon_{2}=b_{2}\left(\varepsilon_{1}\right)\right\}$.

6. Перестройки фазовых портретов в $U$. Множества $\mathrm{B}_{k}$ уже были определены выше. Множества $\mathrm{E}_{k}$ определим согласно формулировке теоремы 1.

Пусть сначала $\varepsilon \in \mathrm{B}_{1}$. В обозначении $\tilde{x}_{-}\left(\varepsilon_{1}\right)$ будем опускать тильду. Так как для $R(\cdot, 0, \varepsilon)$, а потому и для $Q^{+}(\cdot, 0, \varepsilon), x_{-}\left(\varepsilon_{1}\right)$ - двукратный нуль, $\operatorname{sgn} Q_{x x}^{\prime \prime}\left(x_{-}\left(\varepsilon_{1}\right), \varepsilon\right)=\operatorname{sgn} R_{x x}^{\prime \prime}\left(x_{-}\left(\varepsilon_{1}\right), \varepsilon\right)=-1$, то $z_{-}(\varepsilon)=\left(x_{-}\left(\varepsilon_{1}\right), 0\right)$ является клювом, входящая сепаратриса которого является траекторией поля $\left.X_{\varepsilon}^{+}\right|_{M_{+}}$. Вследствие (12) особая точка $z_{+}(\varepsilon)=\left(x_{+}\left(\varepsilon_{1}\right), 0\right)$ - квазиседло. Из (11) и поведения траекторий в точках $\partial U$ следует, что входящая сепаратриса клюва пересекает $\partial U$ в точке $N^{+}(\varepsilon) \in \gamma_{1}^{+}$, одна входящая (выходящая) сепаратриса квазиседла не выходит из $M_{+}$и пересекает $\partial U$ в точке $N_{i n}^{+}(\varepsilon) \in \gamma_{1}^{+}\left(N_{\text {ext }}^{+}(\varepsilon) \in \gamma_{\text {ext }}^{+}\right)$. Обозначим $L^{+}(\varepsilon), L_{i n}^{+}(\varepsilon)$, $L_{\text {ext }}^{+}(\varepsilon)$ соответственно дуги траекторий векторного поля $X_{\varepsilon}^{+} \mid M_{+}$между точками $z_{-}(\varepsilon)$ и $N^{+}(\varepsilon), z_{+}(\varepsilon)$ и $N_{i n}^{+}(\varepsilon), z_{+}(\varepsilon)$ и $N_{\text {ext }}^{+}(\varepsilon)$. Тогда $L^{-}(\varepsilon)=I\left(L^{+}(\varepsilon)\right), L_{\text {ext }}^{-}(\varepsilon)=I\left(L_{\text {in }}^{+}(\varepsilon)\right)$ и $L_{\text {in }}^{-}(\varepsilon)=I\left(L_{\text {ext }}^{+}(\varepsilon)\right)$ соответственно дуги выходящей сепаратрисы клюва, выходящей и входящей сепаратрисы квазиседла. Через каждую точку множества $U \backslash\left(L^{+}(\varepsilon) \cup L^{-}(\varepsilon) \cup L_{i n}^{+}(\varepsilon) \cup L_{\text {in }}^{-}(\varepsilon) \cup L_{\text {ext }}^{+}(\varepsilon) \cup L_{\text {ext }}^{-}(\varepsilon)\right)$ проходит единственная траектория поля $X_{\varepsilon}$, выходящая из $U$, как при возрастании, так и при убывании времени.

Пусть $\varepsilon^{\prime}, \varepsilon^{\prime \prime} \in \mathrm{B}_{1}$. Покажем, что векторные поля $\left.X_{\mathcal{\varepsilon}^{\prime}}\right|_{U}$ и $\left.X_{\varepsilon^{\prime \prime}}\right|_{U}$ топологически эквивалентны, то есть существует гомеоморфизм $U$, переводящий траектории поля $\left.X_{\varepsilon^{\prime}}\right|_{U}$ в траектории поля $\left.X_{\varepsilon^{\prime \prime}}\right|_{U}$. Достаточно построить гомеоморфизм $h: \bar{U}_{+} \rightarrow \bar{U}_{+}$, где $U_{+}:=U \cap M_{+}$, переводящий особые точки в особые точки и любую траекторию поля $\left.X_{\varepsilon^{\prime}}\right|_{U_{+}}$в траекторию поля $\left.X_{\varepsilon^{\prime \prime}}\right|_{U_{+}}$. Обозначим $S(\varepsilon):=L^{+}(\varepsilon) \cup L_{i n}^{+}(\varepsilon) \cup L_{\text {ext }}^{+}(\varepsilon)$. Ясно, что существует гомеоморфизм $h_{S}: S\left(\varepsilon^{\prime}\right) \rightarrow S\left(\varepsilon^{\prime \prime}\right)$, переводящий точки $z_{-}\left(\varepsilon^{\prime}\right)$ и $z_{+}\left(\varepsilon^{\prime}\right)$ соответственно в точки 
$z_{-}\left(\varepsilon^{\prime \prime}\right)$ и $z_{+}\left(\varepsilon^{\prime \prime}\right)$, дуги $L^{+}\left(\varepsilon^{\prime}\right), L_{i n}^{+}\left(\varepsilon^{\prime}\right), L_{\text {ext }}^{+}\left(\varepsilon^{\prime}\right)$ соответственно в дуги $L^{+}\left(\varepsilon^{\prime \prime}\right), L_{i n}^{+}\left(\varepsilon^{\prime \prime}\right), L_{\text {ext }}^{+}\left(\varepsilon^{\prime \prime}\right)$. Обозначим $Q(\varepsilon)$ связную компоненту $U_{+} \backslash S(\varepsilon)$, в границу которой входят дуги $L^{+}(\varepsilon), L_{i n}^{+}(\varepsilon)$, дуга $l(\varepsilon) \subset \gamma_{1}^{+}$между точками $N^{+}(\varepsilon)$ и $N_{i n}^{+}(\varepsilon)$, а также дуга $y=0, x_{-}\left(\varepsilon_{1}\right) \leq x \leq x_{+}\left(\varepsilon_{1}\right)$. Пусть $z=\zeta(t, x, \varepsilon)$ - уравнение траектории поля $X_{\varepsilon}^{+}$, начинающейся в точке $z=(x, 0): \zeta(0, x, \varepsilon)=z$. Так как имеет место (11), а поле $X_{\varepsilon}^{+}$трансверсально дуге $\gamma_{1}^{+}$, то из $\left[1\right.$, п. 3.6] следует, что существует такая непрерывная функция $T_{\varepsilon}:\left[x_{-}\left(\varepsilon_{1}\right), x_{+}\left(\varepsilon_{1}\right)\right] \rightarrow(-\infty, 0)$, что $\zeta\left(T_{\varepsilon}(x), x, \varepsilon\right) \in l(\varepsilon)$, а $Q(\varepsilon)=\left\{z=\zeta(t, x, \varepsilon): x_{-}\left(\varepsilon_{1}\right)<x<x_{+}\left(\varepsilon_{1}\right), T_{\varepsilon}(x)<t \leq 0\right\}$. Гомеоморфизм $h_{S}: S\left(\varepsilon^{\prime}\right) \rightarrow S\left(\varepsilon^{\prime \prime}\right)$ индуцирует такие гомеоморфизмы $\tau_{ \pm}:[0,1] \rightarrow[0,1], \tau_{ \pm}(0)=0$, что

$$
\forall s \in[0,1] h_{S} \zeta\left(s T_{\varepsilon}\left(x_{ \pm}\left(\varepsilon_{1}^{\prime}\right)\right), x_{ \pm}\left(\varepsilon_{1}^{\prime}\right), \varepsilon^{\prime}\right)=\zeta\left(\tau_{ \pm}(s) T_{\varepsilon}\left(x_{ \pm}\left(\varepsilon_{1}^{\prime \prime}\right)\right), x_{ \pm}\left(\varepsilon_{1}^{\prime \prime}\right), \varepsilon^{\prime \prime}\right)
$$

Пусть $\varphi:\left[x_{-}\left(\varepsilon_{1}^{\prime}\right), x_{+}\left(\varepsilon_{1}^{\prime}\right)\right] \rightarrow\left[x_{-}\left(\varepsilon_{1}^{\prime \prime}\right), x_{+}\left(\varepsilon_{1}^{\prime \prime}\right)\right]-$ гомеоморфизм, $\varphi\left(x_{-}\left(\varepsilon^{\prime}\right)\right)=x_{-}\left(\varepsilon^{\prime \prime}\right)$,

$$
\tau(s, x):=\frac{x_{+}\left(\varepsilon^{\prime}\right)-x}{x_{+}\left(\varepsilon^{\prime}\right)-x_{-}\left(\varepsilon^{\prime}\right)} \tau_{-}(s)+\frac{x-x_{-}\left(\varepsilon^{\prime}\right)}{x_{+}\left(\varepsilon^{\prime}\right)-x_{-}\left(\varepsilon^{\prime}\right)} \tau_{+}(s) .
$$

Равенство $\left.h_{Q}\left(\zeta\left(s T_{\varepsilon^{\prime}}(x), x, \varepsilon^{\prime}\right)\right)=\zeta\left(\tau(s, x) T_{\varepsilon^{\prime \prime}}(\varphi(x)), \varphi(x), \varepsilon^{\prime \prime}\right)\right)$ задает гомеоморфизм $h_{Q}: \overline{Q\left(\varepsilon^{\prime}\right)} \rightarrow \overline{Q\left(\varepsilon^{\prime \prime}\right)}$, переводящий траектории поля $\left.X_{\varepsilon^{\prime}}\right|_{U_{+}}$в траектории поля $\left.X_{\varepsilon^{\prime \prime}}\right|_{U_{+}}$и совпадающий с $h_{S}$ в граничных точках $\overline{Q\left(\varepsilon^{\prime}\right)}$. Аналогично задаются гомеоморфизмы $h_{Q}: \overline{Q\left(\varepsilon^{\prime}\right)} \rightarrow \overline{Q\left(\varepsilon^{\prime \prime}\right)}$ для остальных компонент $Q\left(\varepsilon^{\prime}\right)$ и $Q\left(\varepsilon^{\prime \prime}\right)$ множеств $U_{+} \backslash S\left(\varepsilon^{\prime}\right)$ и $U_{+} \backslash S\left(\varepsilon^{\prime \prime}\right)$. Тогда гомеоморфизм $h: \bar{U}_{+} \rightarrow \bar{U}_{+}$задается равенствами $h(z):=h_{Q}(z)$, для любой компоненты $Q=Q\left(\varepsilon^{\prime}\right)$ множества $U_{+} \backslash S\left(\varepsilon^{\prime}\right)$, содержащей в своем замыкании точку $z$.

Случай $\varepsilon \in \mathrm{B}_{3}$ рассматривается аналогично случаю $\varepsilon \in \mathrm{B}_{1}$. Вместо (11) и (12) надо использовать (13) и (14).

Пусть $\varepsilon \in \mathrm{E}_{1}$. Векторное поле $X_{\varepsilon}$ имеет два квазиседла $z_{ \pm}(\varepsilon)=\left(x_{ \pm}(\varepsilon), 0\right)$ и квазицентр $z_{0}(\varepsilon)=\left(x_{0}(\varepsilon), 0\right)$. Из (16) и (27) имеем $Y\left(x, x_{+}(\varepsilon), 0, \varepsilon\right)>0$ при всех $x \in\left[-d_{2}, d_{2}\right], x \neq x_{+}(\varepsilon)$, а также $Y\left(q(\varepsilon), x_{-}(\varepsilon), 0, \varepsilon\right)=$ $0, Y\left(x, x_{-}(\varepsilon), 0, \varepsilon\right)>0$ при $x \in\left(-d_{2}, q(\varepsilon)\right), x \neq x_{-}(\varepsilon)$ где $x_{0}(\varepsilon)<q(\varepsilon)<x_{+}(\varepsilon)$. Отсюда получаем следующие утверждения: Уравнение $y=Y\left(x, x_{+}(\varepsilon), 0, \varepsilon\right)$ при $x \in\left(-d_{2}, x_{+}(\varepsilon)\right]$ (при $\left.x \in\left[x_{+}(\varepsilon), d_{2}\right)\right)$ задает входящую (выходящую) сепаратрису $L_{+, \text {in }}^{+}(\varepsilon)\left(L_{+, \text {ext }}^{+}(\varepsilon)\right)$ квазиседла $z_{+}(\varepsilon) ; L_{+, \text {in }}^{-}(\varepsilon)=I\left(L_{+, \text {ext }}^{+}(\varepsilon)\right)\left(L_{+, \text {ext }}^{-}(\varepsilon)=I\left(L_{+, \text {in }}^{+}(\varepsilon)\right)\right)$ также входящая (выходящая) сепаратриса $z_{+}(\varepsilon)$. Уравнение $y=Y\left(x, x_{-}(\varepsilon), 0, \varepsilon\right)$ при $x \in\left(-d_{2}, x_{-}(\varepsilon)\right]$ задает входящую сепаратрису $L_{-, \text {in }}^{+}(\varepsilon)$ квазиседла $z_{-}(\varepsilon) ; L_{-, \text {ext }}^{-}(\varepsilon)=I\left(L_{-, i n}^{+}(\varepsilon)\right)$ - выходящая сепаратриса точки $z_{-}(\varepsilon)$. Уравнение $y=Y\left(x, x_{-}(\varepsilon), 0, \varepsilon\right)$ при $x \in\left[x_{-}(\varepsilon), q(\varepsilon)\right]$ задает дугу $L_{-, \text {ext }}^{+}(\varepsilon)$ выходящей сепаратрисы квазиседла $z_{-}(\varepsilon) ; L_{-, \text {in }}^{-}(\varepsilon)=I\left(L_{-, \text {ext }}^{+}(\varepsilon)\right)$ - дуга входящей сепаратрисы $z_{-}(\varepsilon)$. «Петля сепаратрисы» $L_{-, \text {ext }}^{+}(\varepsilon) \cup L_{-, \text {in }}^{-}(\varepsilon)$ является периодической траекторией поля $X_{\varepsilon}$. Все траектории, проходящие через точки кольцевой области $K(\varepsilon)$, ограниченной этой петлей и квазицентром $z_{0}(\varepsilon)$, также периодические. Через каждую точку множества

$$
D(\varepsilon):=U \backslash\left(\overline{K(\varepsilon)} \cup L_{+, \text {in }}^{+}(\varepsilon) \cup L_{+, \text {in }}^{-}(\varepsilon) \cup L_{+, \text {ext }}^{+}(\varepsilon) \cup L_{+, \text {ext }}^{-}(\varepsilon) \cup L_{-, \text {in }}^{+}(\varepsilon) \cup L_{-, \text {ext }}^{-}(\varepsilon)\right)
$$

проходит единственная траектория поля $X_{\mathcal{\varepsilon}}$, выходящая из $U$, как при возрастании, так и при убывании времени.

Случай $\varepsilon \in \mathrm{E}_{2}$ отличается от случая $\varepsilon \in \mathrm{E}_{1}$ только сменой ролей точек $z_{-}(\varepsilon)$ и $z_{+}(\varepsilon)$. Теперь совпадают, образуя петлю, выходящая и выходящая сепаратрисы точки $z_{+}(\varepsilon)$.

В случае $\varepsilon \in \mathrm{B}_{2}$ из (16) и (27) получаем $Y\left(x, x_{-}(\varepsilon), 0, \varepsilon\right)=Y\left(x, x_{+}(\varepsilon), 0, \varepsilon\right)>0$ при всех $x \in\left[-d_{2}, d_{2}\right], x \neq$ $x_{ \pm}(\varepsilon)$. Тем самым, выходящая сепаратриса $L_{-, \text {ext }}^{+}(\varepsilon)$ точки $z_{-}(\varepsilon)$, задаваемая уравнением $y=Y\left(x, x_{-}(\varepsilon), 0, \varepsilon\right)$, $x \in\left[x_{-}(\varepsilon), x_{+}(\varepsilon)\right]$ совпадает с входящей сепаратрисой точки $z_{+}(\varepsilon)$, а входящая сепаратриса $L_{-, i n}^{-}(\varepsilon)=$ $I\left(L_{-, \text {ext }}^{+}(\varepsilon)\right)$ точки $z_{-}(\varepsilon)$ совпадает с входящей сепаратрисой точки $z_{+}(\varepsilon)$. Замкнутая кривая $L_{-, \text {ext }}^{+}(\varepsilon) \cup$ $L_{-, \text {in }}^{-}(\varepsilon)$ является периодической траекторией. Все траектории, проходящие через точки кольцевой области $K(\varepsilon)$, ограниченной этой кривой и квазицентром $z_{0}(\varepsilon)$, также периодические. Сепаратрисы $L_{-, i n}^{+}(\varepsilon)$, $L_{-, \text {ext }}^{-}(\varepsilon), L_{+, \text {ext }}^{+}(\varepsilon)$ и $L_{+, \text {in }}^{-}(\varepsilon)$ определяются также как и в случае $\varepsilon \in \mathrm{B}_{1}$. Через каждую точку множества $D(\varepsilon)$ проходит единственная траектория поля $X_{\varepsilon}$, выходящая из $U$, как при возрастании, так и при убывании времени.

При $\varepsilon=\left(\varepsilon_{1}, 0\right) \in \mathrm{E}_{3}$ точка $x=0$ - нуль функции $R(\cdot, 0, \varepsilon)$ и $R_{x}^{\prime}(0,0, \varepsilon)=-\varepsilon_{1}>0$. Ввиду (8) других нулей на $\left[-d_{2}, d_{2}\right]$ у $R(\cdot, 0, \varepsilon)$ нет. Так как $\mathrm{E}_{3}-$ связное множество, то $\forall \varepsilon \in \mathrm{E}_{3} R(\cdot, 0, \varepsilon)$ имеет на $\left[-d_{2}, d_{2}\right]$ единственный нуль $x_{0}(\varepsilon)$, при этом $R_{x}^{\prime}\left(x_{0}(\varepsilon), 0, \varepsilon\right)>0$. Тем самым, поле $X_{\varepsilon}$ имеет в $U$ единственную особую точку - квазиседло $z_{0}(\varepsilon)=\left(x_{0}(\varepsilon), 0\right)$.

Вследствие (2) и (7) $R(\cdot, 0,0)$ имеет на $\left[-d_{2}, d_{2}\right]$ единственный нуль $x=0$ и $\forall x \in\left[-d_{2}, d_{2}\right] \operatorname{sgn} R(x, 0,0)=$ $\operatorname{sgn} x$. Поэтому поле $X_{0}$ имеет в $U$ единственную особую точку $O$, а поведение его траектории в $U$ аналогично поведению траекторий векторных полей $X_{\varepsilon}, \varepsilon \in \mathrm{E}_{3}$.

Топологическая эквивалентность векторных полей $\left.X_{\varepsilon^{\prime}}\right|_{U}$ и $\left.X_{\varepsilon^{\prime \prime}}\right|_{U}$ при $\varepsilon_{1}^{\prime}$ и $\varepsilon_{1}^{\prime \prime}$, принадлежащих соответственно $\mathrm{B}_{2}, \mathrm{~B}_{3}, \mathrm{E}_{1}, \mathrm{E}_{2}, \mathrm{E}_{3}$, доказывается аналогично тому, как это было сделано для случая $\varepsilon^{\prime}, \varepsilon^{\prime \prime} \in \mathrm{B}_{1}$.

На этом доказательство теоремы 1 закончено. 


\section{Список литературы}

1. Андронов А. А., Леонтович Е. А., Гордон И. И., Майер А. Г. 1966. Качественная теория динамических систем второго порядка. М., Наука. 568.

2. Вирченко Ю. П., Субботин А. В. 2015. Обратимые в широком смысле динамические системы. Науч. ведомости БелГУ. Сер. Математика. Физика. 11: 89-96.

3. Лерман Л. М., Тураев Д. В. 2012. О бифуркациях потери симметрии в обратимых системах. Нелинейная динамика. 8 (2): 323-343.

4. Ройтенберг В. Ш. 2017. О бифуркациях в окрестности особой точки типа «трехкратный сшитый фокус». Известия высших учебных заведений. Поволжский регион. Физ-мат. науки. 2: 18-31. DOI: 10.21685/2072-3040-2017-2-2.

5. Ройтенберг В. Ш. 2020. Локальные бифуркации обратимых кусочно-гладких динамических систем на плоскости. Математика и математическое моделирование. 1: 1-15. DOI: 10.24108/mathm.0120.0000 213

6. Ройтенберг В. Ш. 2020. О некоторых бифуркациях обратимых кусочно-гладких динамических систем на плоскости. Вестник Адыгейского государственного университета. Серия: Естественноматематические и технические науки. 2: 11-17.

7. Филиппов А. Ф. 1985. Дифференциальные уравнения с разрывной правой частью. М., Наука. 224.

8. Хартман Ф. 1970. Обыкновенные дифференциальные уравнения. М., Мир. 720.

9. Guardia M., Seara T. M., Teixeira M. A. 2011. Generic bifurcations of low codimension of planar Filippov systems. J. Differential Equations. 250(4): 1967-2023. DOI: 10.1016/j.jde.2010.11.0163.

10. Han M., Zhang W. 2010. On Hopf bifurcation in non-smooth planar systems. J. Differential Equations. 248: 2399-2416. DOI: 10.1016/j.jde.2009.10.002

11. Hogan S. J., Homer M. E., Jeffrey M. R., Szalai R. 2016. Piecewise Smooth Dynamical Systems Theory: The Case of the Missing Boundary Equilibrium Bifurcations. J. Nonlinear Sci. 26: 1161-1173. DOI 10.1007/s00332016-9301-1

12. Kuznetsov Yu. A. Rinaldi S., Gragnani A. 2003. One-parameter bifurcations in planar Filippov systems. Intern. J. of Bifurcation and Chaos. 13(8): 2157-2188. DOI: 10.1142/S0218127403007874

13. Lamb J. S. W., Roberts J. A. G. 1998. Time-reversal symmetry in dynamical systems: A survey. Physica D. $112(1-2)$ : 1-39. DOI: 10.1016/S0167-2789(97)00199-1

14. Sevryuk M. B. 1986. Reversible systems. Lecture Notes in Math., vol. 1211. Berlin, Springer: 319.

15. Teixeira M. A. 1997. Singularities of reversible vector fields. Physica D. 1-2: 101-118. DOI: 10.1016/ S01672789(96)00183-2

\section{References}

1. Andronov A. A., Leontovich E. A., Gordon I. I., Maier A. G. 1966. Kachestvennaja teorija dinamicheskih sistem vtorogo porjadka [The qualitative theory of dynamical systems of second order]. Moscow, Nauka Publ. 568 (in Russian).

2. Virchenko Yu. P., Subbotin A. V. 2015. Reversible systems in wide sense. Belgorod State University Scientific Bulletin. Ser. Mathematics. Physics. 11: 89-96 (in Russian).

3. Lerman L. M., Turaev D. V. 2012. On symmetry breaking bifurcations in reversible systems. Russian J. Nonlinear Dynamics. 8( 2): 323-343 (in Russian).

4. Roitenberg V.Sh. 2017. On bifurcations in the neighborhood of a singular point of the triple sewn focus type. University proceedings. Volga region. Physical and mathematical siences. 2 (42): 18-31 (in Russian). DOI: $10.21685 / 2072-3040-2017-2-2$.

5. Roitenberg V.Sh. 2020. Local bifurcations of reversible piecewise smooth planar dynamical systems. Mathematics and mathematical modelling. 1: 1-15 (in Russian). DOI: 10.24108/mathm.0120.0000213 
6. Roitenberg V.Sh. 2020. On some bifurcations of reversible piecewise smooth planar dynamical systems. Bulletin of Adyghe State Universiry. Ser. "Natural-Mathematical and Technical Sciences". 2: 11-17 (in Russian).

7. Filippov A.F. 1985. Differencial'nye uravnenija s razryvnoj pravoj chast'ju [Differential equations with a discontinuous right-hand side]. Moscow, Nauka Publ. 224 (in Russian).

8. Hartman. Ph. 1970. Obyknovennye differencial'nye uravneniya [Ordinary differential equations] Moscow, Mir Publ. 720 (in Russian).

9. Guardia M., Seara T.M., Teixeira M.A. 2011. Generic bifurcations of low codimension of planar Filippov systems. J. Differential Equations. 250(4): 1967-2023. DOI: 10.1016/j.jde.2010.11.0163.

10. Han M., Zhang W. 2010. On Hopf bifurcation in non-smooth planar systems. J. Differential Equations. 248: 2399-241. DOI: 10.1016/j.jde.2009.10.002

11. Hogan S. J., Homer M. E., Jeffrey M. R., Szalai R. 2016. Piecewise Smooth Dynamical Systems Theory: The Case of the Missing Boundary Equilibrium Bifurcations. J. Nonlinear Sci. 26: 1161-1173. DOI 10.1007/s00332016-9301-1

12. Kuznetsov Yu.A. Rinaldi S., Gragnani A. 2003. One-parameter bifurcations in planar Filippov systems. Intern. J. of Bifurcation and Chaos. 13(8): 2157-2188. DOI: 10.1142/S0218127403007874

13. Lamb J.S.W., Roberts J.A.G. 1998. Time-reversal symmetry in dynamical systems: A survey. Physica D. 112(1-2): 1-39. DOI: 10.1016/S0167-2789(97)00199-1

14. Sevryuk M. B. 1986. Reversible systems. Lecture Notes in Math., vol. 1211. Berlin, Springer: 319.

15. Teixeira M. A. 1997. Singularities of reversible vector fields. Physica D. 1-2: 101-118. DOI: 10.1016/ S01672789(96)00183-2

Конфликт интересов: о потенциальном конфликте интересов не сообщалось.

Conflict of interest: no potential conflict of interest related to this article was reported.

Получена 21.11.2021

\section{СВЕДЕНИЯ ОБ АВТОРЕ}

Ройтенберг Владимир Шлеймович - кандидат физико-математических наук, доцент, доцент кафедры высшей математики Ярославского государственного технического университета

http://orcid.org/0000-0002-1293-7998

Московский проспект, 88, Ярославль, 150023, Россия

E-mail: vroirenberg@mail.ru

INFORMATION ABOUT THE AUTHOR

Vladimir Roytenberg - PhD in Physics and Mathematics, Associate Professor, Associate Professor of the Department of Higher Mathematics, Yaroslavl State Technical University, Yaroslavl, Russia 